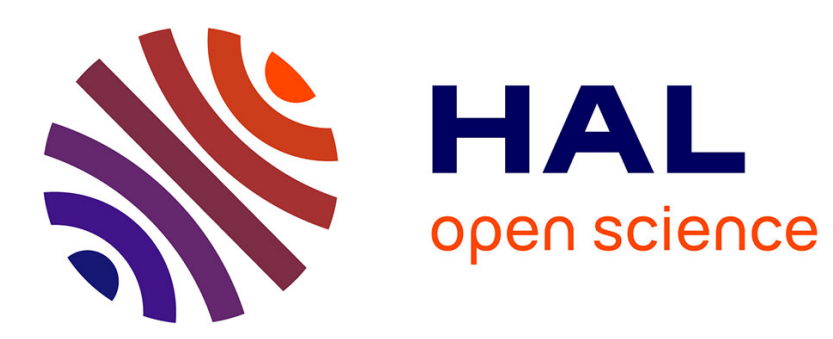

\title{
Pluralité des ressources pour l'action dans les processus de transition formation-emploi chez les enseignants du secondaire
}

\author{
Thérèse Perez-Roux, Xavière Lanéelle
}

\section{- To cite this version:}

Thérèse Perez-Roux, Xavière Lanéelle. Pluralité des ressources pour l'action dans les processus de transition formation-emploi chez les enseignants du secondaire. Les Sciences de l'éducation pour l'ère nouvelle: revue internationale, 2015, 4/2015, pp.17-42. 10.3917/lsdle.484.0017 . hal-01518479

\author{
HAL Id: hal-01518479 \\ https://hal.science/hal-01518479
}

Submitted on 3 Mar 2018

HAL is a multi-disciplinary open access archive for the deposit and dissemination of scientific research documents, whether they are published or not. The documents may come from teaching and research institutions in France or abroad, or from public or private research centers.
L'archive ouverte pluridisciplinaire HAL, est destinée au dépôt et à la diffusion de documents scientifiques de niveau recherche, publiés ou non, émanant des établissements d'enseignement et de recherche français ou étrangers, des laboratoires publics ou privés. 
Perez-Roux, T et Lanéelle, X. (2015). Pluralité des ressources pour l'action dans les processus de transition formation-emploi chez les enseignants du secondaire. Les Sciences de l'Education pour l'ère nouvelle, 48(4), pp. 17-40. https://www.cairn.info/revue-les-sciences-de-1-education-pour-1-ere-nouvelle-2015-4-p-17.htm

\title{
Pluralité des ressources pour l'action dans les processus de transition formation-emploi chez les enseignants du secondaire
}

\author{
Thérèse Perez-Roux ${ }^{*} \&$ Xavière Lanéelle ${ }^{* *}$ \\ * LIRDEF, EA 3749, Université de Montpellier 3. \\ ** CREN, EA 2661, Université de Nantes.
}

\begin{abstract}
Résumé
La recherche s'inscrit dans une perspective sociologique à visée compréhensive. Elle s'intéresse aux processus de construction de la professionnalité enseignante, aux dynamiques d'insertion professionnelle et aux ressources plurielles mobilisées par les néo-enseignants du secondaire. La méthodologie de l'enquête est double: quantitative avec un questionnaire mis en ligne entre février et avril $2013(\mathrm{n}=160)$, qualitative avec 12 entretiens semi-directifs conduits avec des fonctionnaires-stagiaires de différentes disciplines. Les résultats mettent en lumière un système de ressources mobilisant normes institutionnelles relayées par la formation initiale et continue, cultures professionnelles plus ou moins partagées et formes de soutien dans lesquelles le rapport au tuteur prend une place essentielle. On constate que «l'idéal du métier » sert à orienter l'activité. Les élèves, bien que parfois déroutants, constituent aussi une ressource essentielle.
\end{abstract}

Mots clés : transition professionnelle, insertion professionnelle, ressources, professionnalisation, contextes.

\begin{abstract}
The research comes within a sociological comprehensive approach. It focuses on the process of teachers' professionality construction, on the professional integration dynamics and on the various resources mobilized by trainees. Survey methodology is dual-framed: quantitative with a questionnaire on line from February to April 2013 ( $\mathrm{n}=160)$, qualitative with 12 semi-structured interviews with trainee teachers of various disciplines. Results highlight a resource system that mobilize institutional norms, relayed by the initial and in-service training, by more or less shared professional cultures and by forms of support in which the relationship to the tutor takes an essential place. It is noticed that "the profession ideal" acts as a guide to the activity. Pupils, disconcerting sometimes, are also a main resource.
\end{abstract}

Key words: Professional transition, professional integration, resources, professionalization, contexts.

\section{Resumen}

El estudio se inscribe en una perspectiva sociológica a finalidad comprensiva. Trata de los procesos de construcción de la profesionalidad docente, de las dinámicas de inserción profesional y de los recursos plurales movilizados por los profesores principiantes del secundario. La recopilación de datos es doble: cuantitativa con un cuestionario en línea entre febrero y abril del 2013 ( $\mathrm{n}=160)$, cualitativa con 12 entrevistas semi-directivas con docentes cursillistas de diferentes disciplinas. Los resultados ponen en evidencia un sistema de recursos que moviliza normas institucionales relevadas por la formación inicial y continua, culturas profesionales más o menos compartidas y formas de apoyo en las cuales la relación con el tutor es esencial. Se observa que " el ideal del oficio " sirve para orientar la actividad. Los alumnos, aunque a veces desconcertantes, constituyen también un recurso esencial.

Palabras claves: transición profesional, Inserción profesional, recursos, profesionalización, contextos 
Perez-Roux, T et Lanéelle, X. (2015). Pluralité des ressources pour l'action dans les processus de transition formation-emploi chez les enseignants du secondaire. Les Sciences de l'Education pour l'ère nouvelle, 48(4), pp. 17-40. https://www.cairn.info/revue-les-sciences-de-1-education-pour-1-ere-nouvelle-2015-4-p-17.htm

\section{Pluralité des ressources pour l'action dans le processus de transition formation-emploi chez les enseignants du secondaire}

\section{Contexte de l'étude : une professionnalisation en mouvement}

L'article s'intéresse aux processus de construction de la professionnalité enseignante, aux dynamiques d'insertion professionnelle et aux ressources plurielles mobilisées par les néoenseignants du secondaire dans un contexte d'universitarisation de la formation.

Depuis la réforme de la formation des enseignants mise en œuvre en $2010^{1}$, les étudiants doivent obtenir un master pour enseigner. Pendant les trois premières années de la réforme durant lesquelles s'est déroulée notre enquête, trois logiques parfois difficiles à articuler sont à l'œuvre: produire un travail de recherche, réussir le concours de recrutement et se professionnaliser. La professionnalisation est alors soutenue par quelques stages d'observation, de pratique accompagnée et, dans le meilleur des cas, par un stage en responsabilité de quelques semaines durant la deuxième année de master.

Devenus fonctionnaires-stagiaires (FS) ${ }^{2}$ à l'issue de l'obtention du master et de la réussite du concours d'enseignement, ils enseignent le plus souvent dans leur région d'origine $(72 \%)$ ou dans une académie limitrophe $(12 \%)^{3}$. Ils dépendent institutionnellement du Rectorat qui peut choisir l'IUFM de la région comme opérateur de la formation complémentaire proposée durant l'année. Les FS entrent ainsi dans une troisième année de professionnalisation : enseignants en responsabilité sur un temps quasi complet, accompagnés par un tuteur sur le terrain, ils bénéficient en 2012-2013 d'une décharge de 3h par semaine pour se former. En ce sens, ils sont à la fois entièrement responsables de leurs enseignements, considérés comme des enseignants à part entière, bien qu'accompagnés sur le terrain par un tuteur expérimenté ${ }^{4}$.

\section{Repères théoriques}

\subsection{Transition formation-emploi}

L'étude s'intéresse aux situations de transition entre formation et entrée dans le métier en mobilisant plusieurs indicateurs que Dupuy et Le Blanc (2001) utilisent pour montrer le caractère hétérogène des situations de transition. Tout d'abord, la transition évoque un processus de développement, vecteur essentiel de changement, qui suppose de prendre en compte les temporalités. L'adaptation des individus au nouvel environnement implique la transformation de certaines valeurs, l'appropriation de nouveaux rôles et à travers eux une modification de l'image de soi, l'acquisition de nouvelles compétences, voire un changement de style de vie. Par la combinaison de cadres de référence, de modes de pensée et d'action anciens et nouveaux, la transition génère des remaniements identitaires.

En même temps, si la transition entraîne souvent un changement, elle ne peut en aucun cas s'y réduire : entre moments de déstabilisation et de relative stabilité, elle articule continuité et

\footnotetext{
${ }^{1}$ Définition des compétences à acquérir par les professeurs, documentalistes et conseillers principaux d'éducation pour l'exercice de leur métier : circulaire $\mathrm{n}^{\circ}$ 2010-105 du 13-7-2010. http://www.education.gouv.fr/pid24256/n-29du-22-juillet-2010.html.

${ }^{2}$ L'appellation fonctionnaire-stagiaire est celle en cours durant l'année 2012-2013.

${ }^{3}$ Ces pourcentages correspondent aux résultats de l'enquête conduite en 2013 dans l'académie de Nantes (Pays de Loire).

${ }^{4}$ Depuis la rentrée 2013, ils passent les épreuves du concours en Master 1 et sont enseignants-stagiaires l'année de M2, affectés à mi-temps dans un établissement scolaire.
} 
Perez-Roux, T et Lanéelle, X. (2015). Pluralité des ressources pour l'action dans les processus de transition formation-emploi chez les enseignants du secondaire. Les Sciences de l'Education pour l'ère nouvelle, 48(4), pp. 17-40. https://www.cairn.info/revue-les-sciences-de-1-education-pour-1-ere-nouvelle-2015-4-p-17.htm

rupture, changement et résistance au changement, combinant de façon dynamique « logiques de scission, d'élimination et de conservation » (Dupuy et Le Blanc, 2001, p. 63).

Enfin, en questionnant les représentations initiales, les logiques d'action antérieures, la transition conduit à des formes de distanciation: de nouvelles orientations individuelles et collectives peuvent être envisagées à partir de situations vécues comme déséquilibrées ou insatisfaisantes. Nécessités et contraintes propres au nouvel environnement poussent les individus et les groupes à développer des stratégies de régulation et d'adaptation.

Cet ensemble d'éléments éclaire le processus formation-emploi que nous étudions ici. De fait, le mouvement de passage que constitue la transition met en jeu des éléments existentiels et professionnels puissants. Pour Zitoun et Perret-Clermont (2001), il suppose appropriation de savoirs nouveaux, socialisation professionnelle et activité de personnalisation permettant de prendre en compte les ancrages personnels mis en tension, les leviers et les obstacles à l'engagement tant personnel que professionnel. Enfin, en prise avec des changements d'ordre professionnel, la période de transition confronte parfois l'individu à des problèmes d'intégration dans la nouvelle communauté socioprofessionnelle, construite sur des systèmes de valeurs, des normes et des rôles spécifiques.

Qu'en est-il pour les enseignants qui s'insèrent dans un métier nécessitant la construction de compétences plurielles attendues par l'institution qui les (en)cadre ${ }^{5}$ ? Quelles ressources doivent-ils mobiliser pour traverser cette phase de transition professionnelle et s'insérer dans leur nouvel univers de travail ?

\subsection{Insertion professionnelle et construction de la professionnalité enseignante}

De nombreux chercheurs s'intéressent aux processus d'insertion des enseignants et à la manière dont ils s'adaptent aux contextes de travail (Hétu, Lavoie et Baillauquès, 1999 ; Rayou et van Zanten, 2004 ; Perez-Roux, 2006 ; Guibert, Lazuech et Rimbert, 2008 ; Wentzel, Akkari, Coen et Changkakoti, 2011; Lanéelle, 2012; Perez-Roux et Lanéelle, 2012). Ces travaux, à orientation sociologique, tentent de comprendre les difficultés, les tensions, les réaménagements nécessaires, voire les stratégies défensives pour faire face aux exigences du métier.

D'autres chercheurs, parmi lesquels Wittorski et Briquet-Duhazé (2008), Ria (2009), PerezRoux (2012a) creusent davantage, à des grains d'analyse plus fins, la manière dont les enseignants débutants ajustent leur activité dans des situations complexes à gérer, et parviennent à développer des pratiques efficientes, qui renforcent leur identité professionnelle. Ainsi les processus de construction de la professionnalité articulent à la fois développement des compétences attendues dans l'exercice d'un métier, prise en compte des normes professionnelles permettant de juger de la qualité et du sens du travail, lesquelles sont souvent spécifiques au métier d'enseignant puisque l'élève est l'objet de ce travail sur autrui, mobilisation de ressources pour/dans l'action, et remaniements identitaires.

\footnotetext{
${ }^{5}$ Au moment de l'étude, le Bulletin Officiel n 29 du 22 juillet 2010 définit les 10 compétences à acquérir par les professeurs pour l'exercice de leur métier : C1 : Agir en fonctionnaire de l'Etat et de façon éthique et responsable ; C2 : Maîtriser la langue française pour enseigner et communiquer ; C3 : Maîtriser les disciplines et avoir une bonne culture générale ; C4 : Concevoir et mettre en œuvre son enseignement ; C5 : Organiser le travail de la classe ; C6 : Prendre en compte la diversité des élèves ; C7 : Evaluer les élèves ; C8 : Maîtriser les technologies de l'information et de la communication ; C9 : Travailler en équipe et coopérer avec les parents et les partenaires de l'école ; C10 : Se former et innover. http://www.education.gouv.fr/pid24256/n-29-du-22-juillet-2010.html. Ce texte a ensuite été abrogé et remplacé par l'arrêté du 1er juillet 2013, BO n 30 du 25 juillet 2013, entré en vigueur au 1er septembre 2013.
} 
Perez-Roux, T et Lanéelle, X. (2015). Pluralité des ressources pour l'action dans les processus de transition formation-emploi chez les enseignants du secondaire. Les Sciences de l'Education pour l'ère nouvelle, 48(4), pp. 17-40. https://www.cairn.info/revue-les-sciences-de-1-education-pour-1-ere-nouvelle-2015-4-p-17.htm

Pour Jorro et de Ketele (2011), si la professionnalité émergente suppose la construction de gestes professionnels en référence aux prescriptions institutionnelles, elle nécessite aussi une appropriation par le sujet qui doit construire le sens de son action.

Notre étude se centre sur ce qui permet aux débutants d'assurer leur(s) tâches, de répondre aux exigences institutionnelles, de s'insérer dans les collectifs et de progresser dans l'exercice du métier. Faire le choix d'entrer par les ressources semble opportun pour comprendre ce qui se joue dans cette phase d'insertion professionnelle. Quels appuis leur permettent de développer les compétences attendues, de s'approprier peu à peu les règles du métier ? De façon plus générale quelles ressources disent-ils mobiliser prioritairement ? Sont-elles aisément identifiables pour l'acteur lui-même comme pour le chercheur?

\subsection{Ressources pour penser/agir et formes de reconnaissance}

Dans son acception générale, les ressources concernent les possibilités d'action d'un individu ou d'un collectif, les moyens intellectuels et matériels, les produits dont il dispose ou dont il peut disposer ; elles intègrent les astuces, les qualités de débrouillardise, les petits arrangements avec soi-même et avec autrui, les repères pris ou construits dans l'environnement et qui vont orienter l'action. Dans le temps de professionnalisation-insertion, les ressources renvoient donc à des potentialités qui pourraient s'activer par le biais de l'expérience et conduire au développement singulier des compétences attendues.

Notre approche consiste à montrer la pluralité des ressources, mobilisées et articulées. Qu'elles soient matérielles (documentation), liées directement ou indirectement à la formation initiale et continue, relationnelles ou individuelles, ces ressources contribuent à la construction de gestes professionnels (Bucheton, 2009) qui inscrivent l'enseignant à la fois dans son activité et dans la communauté des enseignants qui le/se reconnaît à travers un certain nombre de valeurs partagées sur des objets professionnels (savoirs, élèves, éducation, métier, etc.).

En ce sens, la reconnaissance est non seulement un atout, puisqu'elle légitime l'acteur aux yeux des autrui significatifs (institution, collègues, élèves, parents), mais elle est aussi essentielle pour soi. Honneth (2000) définit le besoin de reconnaissance à partir de trois principes qui forment la grammaire de notre vie sociale : amour ou sollicitude personnelle ; considération et respect ; estime et reconnaissance de l'utilité de chacun qui fondent les solidarités de groupes. Comment et dans quelle mesure ces éléments participent-ils des processus d'insertion professionnelle dans le temps de transition formation-emploi ?

Dans notre étude, les FS ont à trouver leur place dans un nouvel univers professionnel, à vivre l'épreuve / le défi du «vrai » métier et à faire leurs preuves. De quelle manière procèdent-ils ? Quelles ressources leurs sont réellement utiles pour penser, mettre en œuvre, réguler et évaluer les apprentissages ? Quels sont leurs atouts pour se faire reconnaitre des élèves et de l'ensemble de la communauté éducative?

\section{Méthode}

Les différentes ressources mentionnées ci-dessus peuvent être mises en relief grâce aux deux volets de l'enquête que nous avons menée.

Le premier volet, situé entre février et avril 2013, a consisté à diffuser un questionnaire auprès de l'ensemble de la population de stagiaires de l'académie de Nantes. L'enquête a été réalisée sur Sphinx 5 et saisie en ligne. Nous choisissons ici de ne traiter que des 160 fonctionnairesstagiaires du second degré, sur les 257 qui y ont répondu (97 FS du premier degré). Cette population est essentiellement féminine (66\%), jeune (89\% des hommes ont moins de 30 ans), même s'il ne faut pas négliger les reconversions ou retours à la vie active (10\%). $74 \%$ des FS sont certifiés, $7 \%$ sont agrégés, $4 \%$ documentalistes, $3 \%$ CPE ; la moitié exerce en collège, $12 \%$ 
Perez-Roux, T et Lanéelle, X. (2015). Pluralité des ressources pour l'action dans les processus de transition formation-emploi chez les enseignants du secondaire. Les Sciences de l'Education pour l'ère nouvelle, 48(4), pp. 17-40. https://www.cairn.info/revue-les-sciences-de-1-education-pour-1-ere-nouvelle-2015-4-p-17.htm

en LEP, le reste en LEGT. Ce sont les enseignants d'EPS, de Langues et de Mathématiques qui forment l'essentiel, respectivement 25, 19 et 16\%, Deux biais possibles doivent néanmoins être évoqués. Dans la mesure où les résultats sont obtenus sur la base d'une auto-déclaration, un biais de désirabilité sociale n'est pas à exclure. De plus, le taux de réponse est plus élevé pour les disciplines où les deux auteurs ont assuré antérieurement des formations.

Par ailleurs, au-delà des questions ouvertes du questionnaire, 12 entretiens semi-directifs ont été conduits avec des FS de différentes disciplines entre janvier et mars 2013: 4 en Éducation physique et sportive (EPS, 1 homme et 3 femmes), 3 en Sciences économiques et sociales (SES, 3 femmes), 3 en Français ( 3 femmes), 2 en Mathématiques ( 2 femmes), âgés de 22 à 29 ans. Seule une partie des données statistiques et des questions est utilisée ici. Celles-ci portent : a) sur les aides ou ressources prioritairement recherchées par les FS ; b) sur les modalités du travail engagé avec le tuteur et les attentes en termes d'accompagnement; c) sur le type de soutien des collègues de l'établissement; d) sur la relation aux élèves; e) sur le rapport à la formation initiale et continue.

Le corpus des entretiens a d'abord été traité à l'aide du logiciel Alceste (Lanéelle \& Perez-Roux, 2014). Une analyse thématique de contenu (verticale et horizontale) a été ensuite réalisée manuellement pour tenter de repérer les différents types de ressources évoqués par les néotitulaires et tenter d'en comprendre les articulations. L'analyse de ces entretiens permet d'étayer certains éléments significatifs recueillis par questionnaire, de les corroborer, les consolider, les relativiser parfois.

\section{Résultats}

\subsection{Des ressources matérielles mobilisées pour assurer les tâches d'enseignement}

Les ressources documentaires apparaissent comme largement prioritaires. Le plus souvent, il est fait référence au manuel (ou à d'autres livres). Les documents numériques (eduscol, cours en ligne, site académique) ainsi que le Bulletin Officiel (BO), les programmes sont aussi mentionnés. " J'ai beaucoup épluché, lu et relu les documents ressources des programmes. J'ai aussi souvent regardé les séquences de cours disponibles sur le site académique de Nantes pour comprendre vers quels objectifs il faut tendre avec les élèves. » $\left(\mathrm{e} 221^{6}\right)$.

Les 12 entretiens livrent des informations plus précises quant aux modalités d'utilisation de ces supports. Les programmes officiels restent un appui indispensable pour plusieurs raisons : recherche de conformité dans une année de validation et cadre indispensable pour envisager son enseignement : "j'essaie de me détailler un peu le programme. Après je compare avec ce que j'ai trouvé dans le programme et ce qu'il y a dans le livre. Bon, généralement ça coïncide à peu près mais y a des fois des petites notions que, qui sont pas indiquées dans le programme mais qu'on doit faire » (Julie, Maths, 23 ans)

Si les manuels, les sites internet (type éduscol, néopass), les forums, sont omniprésents dans les réponses, les néo-enseignants interrogés expliquent aussi comment ils bricolent ces ressources pour en faire de véritables appuis qui aient un sens pour eux et répondent, au moins en partie, aux exigences institutionnelles. A partir de recherches plurielles, Annie (Français, 23 ans) opère un travail d'appropriation : "pendant l'été j'ai acheté plein de manuels, j'en consulte aussi sur internet, des manuels en ligne [...] Je télécharge des séquences aussi, je vais sur plein de sites.

\footnotetext{
${ }^{6}$ Le code (e221) correspond à l'énoncé du $221{ }^{\text {ème }}$ enquêté ayant répondu à une question ouverte du questionnaire en ligne. A noter que les $160 \mathrm{FS}$ du secondaire se répartissent dans l'ensemble du groupe des répondants $(\mathrm{n}=257)$ qui intègre des enseignants du primaire. Lorsque nous citons des extraits d'entretien, nous indiquerons le nom, la discipline et, à la première citation, l’âge de l'enseignant.
} 
Perez-Roux, T et Lanéelle, X. (2015). Pluralité des ressources pour l'action dans les processus de transition formation-emploi chez les enseignants du secondaire. Les Sciences de l'Education pour l'ère nouvelle, 48(4), pp. 17-40. https://www.cairn.info/revue-les-sciences-de-1-education-pour-1-ere-nouvelle-2015-4-p-17.htm

J'ai toute une liste hein où je vais voir souvent ce qu'il y a, et puis après je fais mon petit mélange en fonction de ce que moi je veux faire... et puis je réadapte mes séquences comme je veux $\gg$.

Luna (Français, 24 ans) dit avoir obtenu « un legs de [sa] tutrice » lors du stage en responsabilité effectué en M2. Toutes les séquences de cette dernière, transmises sur une clé USB et prêtes à l'emploi, constituent une ressource que Luna utilise au gré des besoins, notamment pour les corpus de textes qui appuient la séquence et restent, de son point de vue, complexes à élaborer.

Les enseignants d'EPS convoquent essentiellement les connaissances et compétences construites en formation initiale dans les différentes Activités physiques, sportives et artistiques (APSA), pointant ici ou là, les difficultés rencontrées lorsque telle ou telle activité est mal maîtrisée ou n'a jamais été pratiquée. Aude (SES, 29 ans) utilise aussi, en les adaptant, certains cours suivis en Master dans le cadre de la préparation du concours. Sylvie (SES, 23 ans) confirme : «c'est vrai que je reprends les classeurs, les différentes activités qu'on a pu proposer... C'est vrai que ça me sert. Les cours que je reprends régulièrement, c'est la didactique ».

Par ailleurs, les analyses mettent en avant des réseaux d'aide entre stagiaires, construits parfois dès la formation initiale : petits conseils et «bons plans », mutualisation de documents pour étayer le travail de préparation, échanges de séquences, etc. montrent bien que les ressources utilisées renvoient inévitablement à la conception - préparation des séances ou des séquences avec, en arrière-plan, le désir d'intéresser les élèves. C'est en ce sens que la formation initiale trouve, aux yeux de certains stagiaires, une place réelle mais difficile à définir. Annie (Français) aborde cet aspect : «construire une séquence ou une séance je pense que si j'avais jamais eu de cours à l'IUFM, je serais un peu plus dans le brouillard il me semble. Après, on se rend pas forcément bien compte ».

Ce rapport à la formation (initiale et continue) comme ressource potentielle lors de l'entrée dans le métier est largement abordé à la fois dans le questionnaire et dans les entretiens.

\subsection{Un rapport à la formation relativement contrasté}

\subsubsection{La formation initiale : une expérience courte, des ressources partielles}

Les réponses à la question : «comment qualifieriez-vous la formation au métier que vous avez reçue ? » sont relativement contrastées : si une moitié des néo-enseignants trouvent la formation initiale «bonne » $(42 \%)$ ou «excellente » $(9 \%)$ en termes de professionnalisation, 39\% la qualifient de « passable » ou de «mauvaise » $(9 \%)$.

Les contenus travaillés en formation initiale et jugés utiles pour le métier (trois réponses possibles) s'organisent de façon prioritaire autour de la «didactique disciplinaire » $(68 \%)$, de la «pédagogie» $(59 \%)$ et de «l'analyse des pratiques de stage» $(48 \%)^{7}$. Les apports en «psychologie » $(18 \%)$ et en « sociologie de l'éducation » $(10 \%)$ sont positionnés en retrait bien que, lors des entretiens, les enseignants évoquent parfois une utilité différée ou une meilleure compréhension, par ces approches, des enjeux qui traversent le monde scolaire et ses acteurs. Certains enseignants, à la marge, mettent en avant la nécessité de connaître l'institution et son fonctionnement, les aspects règlementaires ou juridiques, mais aussi les possibilités de mutations et les perspectives de carrière, sur lesquels ils se sentent peu informés.

Les entretiens corroborent ces résultats nuancés. Les trois enseignantes de Français interrogées insistent sur les préparations de séquences qui constituent à présent de réelles ressources. Comme le dit Annie : «il y a des petites méthodes vues en M2 qui peuvent revenir, même la conception : trouver les objectifs, trouver les problématiques, ce genre de choses quoi !». Pour

\footnotetext{
${ }^{7}$ Lorsque l'on ne retient que les réponses classées en rang 1 (total des réponses sur base 100\%), les mêmes items apparaissent mais dans un ordre différent : didactique disciplinaire (50\%); analyse de pratiques de stage (30\%) et pédagogie (17\%).
} 
Perez-Roux, T et Lanéelle, X. (2015). Pluralité des ressources pour l'action dans les processus de transition formation-emploi chez les enseignants du secondaire. Les Sciences de l'Education pour l'ère nouvelle, 48(4), pp. 17-40. https://www.cairn.info/revue-les-sciences-de-1-education-pour-1-ere-nouvelle-2015-4-p-17.htm

Luna, la formation initiale a permis de « prendre du recul et de développer l'esprit critique » tout en abordant la construction concrète de séquences «validées » par une formatrice, expérimentées en stage et analysées a posteriori; ce travail peut désormais être réinvesti et remanié en fonction du contexte d'intervention : « je m'en sers encore aujourd'hui de mes séquences justement. Je les reprends mais je les change. Ça m'a beaucoup aidée pour commencer ». Sarah souligne l'apport de l'observation puis de la mise en situation d'enseignement dans le cadre des stages, et, en même temps, la réflexion sur la transposition didactique, sur les choix de corpus de textes ou sur les modalités d'évaluation qui constituent de réels appuis.

Aude (SES) dit avoir apprécié le travail didactique mais se trouve désormais sous pression temporelle et regrette de ne pouvoir davantage approfondir/améliorer les contenus, faire des recherches documentaires. Considérant la pré-professionnalisation comme un lieu d'expérience et de construction de ressources, cette enseignante revient sur les cours de psycho-pédagogie en Master, qu'elle juge «très formateurs pour débuter dans le métier ». Ces contenus sont venus renforcer son expérience (5 ans) d'assistante d'éducation qui lui ont donné une première approche des élèves, «à recalibrer une fois qu'on est enseignant » au regard de la spécificité de la mission.

Pour d'autres enseignants, la professionnalisation semble ne pas avoir réellement commencé ou avoir été largement minorée en raison des enjeux de réussite au concours. Julie (Mathématiques) : " on a juste fait une préparation au concours. Y avait pas de préparation au métier... et les suivis de stages, on les avait par session de 4 heures où on parlait du stage et du coup forcément du métier un petit peu [...] Je pense que la formation n'a pas servi à grandchose et je ressens pas les effets [...] On se plaignait tous du manque de formation mais en même temps, fallait préparer le concours. Ça prenait déjà tellement de temps... où est-ce qu'on aurait casé cette formation?»

A contrario, la pré-professionnalisation progressive durant la formation initiale en Sciences et techniques des activités physiques et sportives (STAPS) est vécue comme un atout majeur par les néo-enseignants d'EPS qui se positionnent de façon décalée vis-à-vis de leurs collègues stagiaires d'autres disciplines, dont ils jugent la préparation pédagogique insuffisante. La diversité des APSA, le fait de les avoir pratiquées, au moins en partie, durant le cursus universitaire constitue aussi un apport essentiel pour entrer dans le métier avec des repères didactiques concrets et transposables en EPS.

Cet aspect est repris dans les questions ouvertes du questionnaire comme l'évoque cette enseignante d'anglais : "Affirmer mon autorité a été plus facile avec l'expérience [courte] que je possédais en arrivant. Lors de mon stage en M2, j'ai eu la chance d'être avec une excellente conseillère pédagogique pendant 5 semaines qui nous a permis (avec mon binôme) de prendre en charge ses classes, nous a donné de nombreux conseils, ce qui me sert tous les jours en cours $\gg(\mathrm{e} 131)$.

Dans la mesure où la formation initiale est perçue comme moyennement orientée sur le métier, excepté en EPS, qu'en est-il du rapport à la formation continue, telle que vécue durant cette année de professionnalisation?

\subsubsection{La formation continue et ses temporalités : urgences et efficacité vs réflexion sur le métier}

Les avis convergent sur la formation proposée durant l'année : priorité au disciplinaire et ouverture mesurée aux problématiques transversales, centrées plus largement sur le métier. Aude (SES) est très satisfaite d'une formation disciplinaire ajustée à la demande : "on voit comment faire un cours, comment bien l'adapter au niveau des élèves ». Les formations intitulées «gestion de classe », organisées par secteurs géographiques (bassins) et regroupant différentes disciplines sont aussi considérées comme des ressources à plusieurs niveaux. D'une 
Perez-Roux, T et Lanéelle, X. (2015). Pluralité des ressources pour l'action dans les processus de transition formation-emploi chez les enseignants du secondaire. Les Sciences de l'Education pour l'ère nouvelle, 48(4), pp. 17-40. https://www.cairn.info/revue-les-sciences-de-1-education-pour-1-ere-nouvelle-2015-4-p-17.htm

part, pour le côté concret : «pour la gestion de classe, on a des cas concrets et puis des gens qui enseignent encore, enfin sur le terrain quoi ! Donc ils peuvent nous dire ce que eux ils font, ce qu'ils feraient. Et là, on a des réponses!» (Annie, Français); d'autre part, pour le côté rassurant procuré par un espace d'expression qui permet de revenir sur les difficultés liées à la réalité du métier : «les formateurs sont vraiment agréables, ils nous racontent leurs anecdotes, on peut parler librement de tout ce qui va, qui va pas » (Julie, Maths), ou à des formes d'étayage partagé : «c'est intéressant de voir d'autres disciplines. On se rend compte qu'en fait dans n'importe quel cours, on a les mêmes difficultés qui reviennent. C'est rassurant !» (Luna, Français). Le plus souvent, les analyses de situations sont jugées favorablement si elles permettent de prendre du recul et de partager des problématiques professionnelles.

Dans les entretiens, plusieurs FS notent une évolution dans leur rapport à la formation en termes de ressources pour l'action. Comme le dit Luna (Français) : «en début d'année, l'urgence, c'était construire des cours pour la rentrée et j'avais l'impression que ce qu'on nous proposait en formation ne servait à rien... Au fur et à mesure des vendredis qui passaient, et ça c'est surtout en module disciplinaire donc sur les Lettres, j'ai senti que les débats qu'on avait nous faisaient vraiment évoluer [...] et j'ai gagné en efficacité, et en confiance aussi. Du coup je pense que ça aide, quand même, beaucoup ». Malgré cette évolution, évoquée par d'autres FS interrogés, les discours mettent en relief des temporalités diverses, faisant dire à Aude (SES) : " pour moi, le transversal c'est pour plus tard ». Ceci laisse à penser que les ressources sont plus ou moins construites/activées en fonction des urgences (construire des cours et les mettre en œuvre dans la classe), ce qui amène à des formes de hiérarchisation de la part des stagiaires.

La hiérarchisation est inversée du côté des FS en EPS, pour lesquels le niveau de développement professionnel semble plus avancé du fait du parcours antérieur plus professionnalisant (cf. Adé, $\mathrm{XXX)}$. Dans l'espace de la formation, ces derniers restent très critiques en raison de leur formation initiale qui, selon eux, leur donne un temps d'avance sur les autres disciplines. Ce positionnement spécifique dans l'espace scolaire est explicité par Fanny (EPS, 22 ans) : «je pense que c'est dû à notre formation en STAPS qui est assez complète. Du coup, il y a beaucoup de choses qu'on a traitées, que les autres disciplines n'ont pas traitées et on est en décalage ». Les FS en EPS ont donc tendance à dénigrer les contenus transversaux destinés à faire réfléchir des enseignants de différentes disciplines sur des thématiques communes : « en formation, on est avec les autres disciplines et c'est exactement la même chose que ce qu'on a vu pour le concours. On revient sur des thèmes : la motivation, l'évaluation. Donc nous, ça nous barbe... enfin on voit pas le lien [avec le stage], ça sert à rien » (Aline, EPS, 22 ans). En termes de ressources, la priorité est donnée aux APSA dans lesquelles chacun tente de se perfectionner pour enrôler les élèves dans l'activité et trouver une forme de légitimité didactique et pédagogique.

Enfin, malgré leur attentes fortes sur la dimension didactique de la formation, certains FS relativisent les ressources apportées par des formateurs trop experts de leur domaine et loin, selon eux, de la réalité des pratiques des débutants : "les formateurs nous parlent de leurs pratiques extraordinaires et ça fait un décalage avec nous!» (Aude, SES). Ce sentiment de ne pas être à la hauteur, de bricoler parfois sans trop approfondir la réflexion, se double paradoxalement d'un inconfort lorsque les formateurs ne donnent pas de réponses tranchées. Annie (Français) le regrette : «souvent à la fin de la journée j'ai toujours pas la réponse à mes questions, et du coup j'ai plutôt l'impression qu'on nous dit ce qu'il faut pas faire, et pas ce qu'il faut faire. Parce qu'il n'y a pas de réponse euh... voilà ! Mais des indices, je sais pas, quelque chose ». Si les échanges entre pairs peuvent donner des pistes, le manque d'approbation du formateur pose problème : «on aimerait savoir ce qu'il faut faire, enfin, pas ce qu'il faut faire ou quoi faire mais un peu quand même ! » (Annie)

Ainsi, bien que certains FS soient sensibles à la construction du métier à partir de questions transversales, les ressources prioritaires renvoient à des contenus très concrets, concernant 
Perez-Roux, T et Lanéelle, X. (2015). Pluralité des ressources pour l'action dans les processus de transition formation-emploi chez les enseignants du secondaire. Les Sciences de l'Education pour l'ère nouvelle, 48(4), pp. 17-40. https://www.cairn.info/revue-les-sciences-de-1-education-pour-1-ere-nouvelle-2015-4-p-17.htm

directement la classe et répondant aux urgences des débuts. L'utilité se perçoit davantage sur des temporalités courtes que dans la durée. Comment cette orientation de la demande est-elle mise en œuvre dans le travail avec les tuteurs en charge de l'accompagnement des néo-enseignants ?

\subsection{L'établissement et ses acteurs : entre étayage direct et indirect}

\subsubsection{Le tuteur au cour du dispositif de professionnalisation ?}

Parmi les 160 FS du secondaire ayant répondu à l'enquête par questionnaire, 94\% ont bénéficié de l'accompagnement d'un tuteur dès la rentrée, $76 \%$ des stagiaires ont un seul tuteur, $22 \%$ en ont deux. Si un fort pourcentage de tuteurs enseigne dans le même établissement (87\%), les $20 \%$ qui travaillent à l'extérieur ne peuvent sans doute pas réaliser le même type de suivi, notamment en termes de connaissance/analyse du contexte local.

Une question portait sur la fréquence des moments de formation. En milieu d'année, $40 \%$ des FS rencontrent leur tuteur en moyenne « une fois par semaine ou plus », 18\% « une fois par quinzaine » et $9 \%$ « une fois par mois ». Enfin, 29\% disent travailler avec lui «à la demande » et $4 \%$ des enseignants du corpus disent avoir « rarement » l'occasion de se former avec lui.

La question suivante : «Sur quoi portent essentiellement vos échanges avec le(s) tuteur(s) ?» (2 réponses possibles) permettait de préciser les objets de travail et de confirmer les ressources jugées essentielles. "Les questions didactiques: construction des séances et des séquences, choix des évaluations, etc. » $(78 \%)$ et «les questions pédagogiques : organisation des élèves dans la classe, régulation des élèves difficiles, distribution de la parole, etc. » (71\%), arrivent largement en tête, laissant loin derrière « les questions liées à l'établissement : organisation des conseils de classe, construction de projets pédagogiques, etc. » $(23 \%)$ ou celles renvoyant aux «relations avec la vie scolaire et avec les parents » $(5 \%)$. Pour $92 \%$ des FS, ces rencontres avec le tuteur répondent aux attentes, même si, comme l'évoque un-e FS : «Soyons honnêtes, quand on débute, on ignore ce dont on a besoin. » (e46). Les résultats permettent de comprendre dans quelle mesure cet accompagnement constitue de réelles ressources pour les enseignants qui débutent.

Les appréciations les plus nombreuses font référence à l'expérience du tuteur qui lui permet de donner des conseils, à son écoute qui rassure, apporte sécurité et confiance : "Ces rencontres permettent une marge de liberté dans l'enseignement tout en ayant un appui sur lequel se référer. »(e6). Comme le développe cette enseignante dans une question ouverte : "Ma tutrice s'est montrée très disponible avec moi, elle a répondu patiemment à toutes mes questions (très nombreuses au début). Elle s'est montrée extrêmement bienveillante, et surtout m'a rassurée au début de l'année, ce dont j'avais le plus besoin, (j'avais l'impression d'être jetée dans le métier sans aucune pratique, hormis une semaine de stage avec un professeur dans le fond de la classe). Nous avons pratiquement la même approche de travail, ce qui nous a permis de créer des séquences en commun. Ce travail de collaboration a été très satisfaisant. Enfin, elle a assisté à de nombreux cours et elle a passé au crible ma pratique (je crois que c'est cela qui m'a le plus aidé à avoir un regard critique sur mon travail). »(e218).

Au moment de l'enquête (deuxième trimestre), les attentes s'inscrivent dans la continuité de ce qui a été initié avec le(s) tuteur(s) et paraît satisfaire les FS. Les savoirs d'expérience des tuteurs, reconnus par les corps d'inspections qui les ont sollicités, ont permis aux FS de s'ancrer dans la réalité du métier. Ces derniers mettent en avant la nécessité d'une aide éventuelle du tuteur si de nouveaux problèmes survenaient d'ici la fin de l'année. Certains expriment une inquiétude quant à leurs capacités et compétences qui semblent encore relever d'équilibres fragiles dans la mesure où ils ne maîtrisent pas tous les paramètres en jeu dans l'acte d'enseignement. Les attentes se situent essentiellement dans le court terme. Pourtant, à la marge des discours, se repèrent des perspectives de progrès dans l'agir professionnel souvent reliées à des idéaux. Ce témoignage emblématique montre l'impact de certains tuteurs dans la 
Perez-Roux, T et Lanéelle, X. (2015). Pluralité des ressources pour l'action dans les processus de transition formation-emploi chez les enseignants du secondaire. Les Sciences de l'Education pour l'ère nouvelle, 48(4), pp. 17-40. https://www.cairn.info/revue-les-sciences-de-1-education-pour-1-ere-nouvelle-2015-4-p-17.htm

construction du soi professionnel : «j'ai envie que l'on garde contact car ils [deux tuteurs] ont vraiment changé ma vie, ils m'ont poussée et maintenant je me sens réellement bien dans ma vie professionnelle. Ce sont deux personnes qui m'auront beaucoup marquée. » (e185).

\subsubsection{Le(s) collectif(s) : normes, usages, formes d'accompagnement}

En termes de ressources relationnelles et organisationnelles, une question revenait sur l'accueil dans l'établissement en début d'année. Parmi les $91 \%$ qui ont bénéficié d'un accueil de l'équipe de direction, $61 \%$ ont été présentés à des collègues de l'établissement. Par ailleurs, au fil de l'année, $97 \%$ des FS disent avoir noué « des relations professionnelles satisfaisantes avec des collègues (tuteur exclu)». Ces interactions semblent renforcer les ressources potentielles et ouvrent sur d'autres questions, finalement peu abordées avec les tuteurs. En effet, à la question : «Pouvez-vous indiquer quelles ressources vous apportent ces collègues?» (2 réponses possibles), les items retenus balaient - au-delà de l'aide documentaire (38 occurrences des réponses à la question ouverte sur ces ressources) - un ensemble de registres jusque-là peu mobilisés ; ainsi, ces autres enseignants apportent « des conseils » $(61 \%)$, « des repères sur le fonctionnement de l'établissement « $(44 \%)$, «des collaborations : partager la préparation de cours, devoirs communs, etc. » $(35 \%)$ et « un soutien moral » $(34 \%)$.

Enfin, le travail en équipe au sein de l'établissement permet de développer de nouvelles ressources, combinées à des formes d'acculturation et de positionnement des néo-enseignants quant à leurs pratiques dans l'espace scolaire. $83 \%$ des FS affirment qu'un travail en équipe existe au sein de leur établissement et ils sont $67 \%$ à y être réellement associés. Les trois réponses possibles rendent compte de modalités diverses : «préparer des séances en commun » ou «développer des collaborations: IDD, $\mathrm{TPE}^{8}$, histoire des arts, etc. » obtiennent respectivement $30 \%$ des scores ; «choisir une progression » $(29 \%)$, " améliorer le suivi des élèves » $(25 \%)$, « préparer des devoirs en commun » $(23 \%)$ attestent de priorités que se donnent les FS ou que les collectifs enseignants proposent pour professionnaliser, d'une autre manière, les entrants dans le métier. Cette dimension, révélée dans le questionnaire, montre l'importance des autres enseignants dans un processus de développement des compétences professionnelles, une sorte d'étayage moins visible mais complémentaire de ce que fait le tuteur, centré davantage sur l'accompagnement en classe. Les entretiens reprennent ces différents éléments : devoirs communs, échanges de supports pour le cours, discussion pour se mettre d'accord, harmonisations pour le brevet blanc sont évoqués comme étayage supplémentaire, de même que se construisent des affinités qui permettent: «de parler d'autre chose, de mettre un peu d'humour $[. .$.$] savoir prendre de la distance » (Luna, Français).$

\subsection{Faire l'expérience des élèves : obstacle et moteur de l'action}

Les enseignants entrant dans le métier véhiculent un certain nombre d'idéaux, construits en amont et/ou pendant la formation. A la question «quels sont les aspects du métier d'enseignant qui vous semblent prioritaires aujourd'hui ?», les premiers choix (parmi 15 items proposés) rendent compte de tendances fortes : «susciter le plaisir d'apprendre » $(25 \%)$; «maitriser et transmettre des savoirs disciplinaires » $(18 \%)$; « veiller à la progression de ses élèves » $(12 \%)^{9}$. De façon complémentaire, l'image d'un enseignant «juste» $(26 \%)$, « disponible » $(16 \%)$, «stimulant» $(13 \%)$ et «rigoureux» $(11 \%)$ donne à comprendre les qualités largement privilégiées.

\footnotetext{
${ }^{8}$ Itinéraires de découverte (IDD) en collège, Travaux personnels encadrés (TPE) au lycée.

${ }^{9} \mathrm{Si}$ on prend en compte l'ensemble des 3 réponses possibles, l'ordre est un peu modifié : « susciter le plaisir d'apprendre » (52\%); "veiller à la progression de ses élèves » (39\%); «maitriser et transmettre des savoirs disciplinaires $\gg(30 \%)$.
} 
Perez-Roux, T et Lanéelle, X. (2015). Pluralité des ressources pour l'action dans les processus de transition formation-emploi chez les enseignants du secondaire. Les Sciences de l'Education pour l'ère nouvelle, 48(4), pp. 17-40. https://www.cairn.info/revue-les-sciences-de-1-education-pour-1-ere-nouvelle-2015-4-p-17.htm

Autour de cet idéal, se construit progressivement un rapport au métier dans lequel les uns ou les autres peuvent vivre des expériences positives. L'analyse des énoncés d'une question ouverte portant sur la «description de moments de réussite » révèle des situations intimement liées au plaisir, à l'intérêt, à l'implication des élèves dans les tâches proposées. Leur participation semble constituer le creuset d'une légitimité et d'une reconnaissance professionnelle encore balbutiante ou à confirmer. De ce point de vue, les retours explicites ou implicites des élèves, leur engagement dans le processus d'apprentissage donne aux débutants l'occasion de se projeter dans l'avenir et de faire le point sur les acquisitions en cours pour eux-mêmes, en apprentissage d'un métier multidimensionnel qui à la fois les éprouve et les fait grandir.

La volonté affirmée de susciter le plaisir d'apprendre place l'élève au cœur des enjeux et met les néo-enseignants face à un défi majeur : intéresser, enrôler dans le travail d'apprentissage et trouver ainsi une forme de légitimité pédagogique et didactique pour les élèves. Cette quête se révèle délicate et les FS expriment un certain nombre de difficultés. Lorsque l'on prend les premières réponses à la question : «dans votre exercice du métier, quelles sont les difficultés rencontrées à ce jour? », ce sont les difficultés «d'ordre pédagogique : gestion de classe difficile, publics scolaires hétérogènes, etc. » $(35 \%)$, «d'ordre didactique : adéquation des contenus aux élèves, choix, progressivité des apprentissages, etc. » (19\%) et celles « liées aux conditions de travail : matériel insuffisant, locaux inadaptés, horaires mal placés, etc. » (13\%) qui constituent les principales sources de tension entre les idéaux et la réalité du métier.

Des moments difficiles vécus durant l'année sont précisés dans une question ouverte intégrée dans le questionnaire; ils portent le plus souvent sur des malentendus ou des conflits entre les élèves, ou entre les élèves et l'enseignant. Une préoccupation récurrente traverse les discours : ces nouveaux enseignants, confrontés à l'hétérogénéité, expriment le désir de faire progresser (tous) leurs élèves mais se trouvent contraints à des ajustements, à des choix qui ne les satisfont pas. L'image de l'enseignant idéal se trouve chahutée. C'est en partie pour cette raison que l'engagement des élèves dans le travail demandé apporte à ces néo-enseignants un réel sentiment de légitimité professionnelle.

Pour autant les FS expriment le sentiment d'avoir progressé (3 réponses possibles) prioritairement sur: «la construction des contenus de séance » (44\%), «l'adaptation des contenus aux élèves » $(37 \%)$ et «la gestion du groupe classe » $(33 \%)$. Mais leurs progrès concernent aussi « la mise en activité/engagement des élèves » $(26 \%)$, « la clarté des consignes données » $(25 \%)$ et de façon plus large : «la planification des séquences sur l'année » $(21 \%)$. Les 6 autres items de la question obtiennent des scores moindres.

Ces résultats sont corroborés au niveau des entretiens. La question de la confiance accordée par les élèves est abordée de façon récurrente dans les discours des FS : «au niveau des méthodes pédagogiques j'aimerais qu'ils pensent que le français c'est un jeu [...] j'ai l'impression que je commence à y arriver et ça me plaît énormément. On a une complicité en fait qui s'est créée, il y a une confiance mutuelle parce que j'ai même confiance en eux, que je pensais pas avoir en commençant, et c'est vraiment merveilleux!» (Luna, Français). Annie exprime cette reconnaissance de la part des élèves avec un grand bonheur : «ils m'ont déjà dit qu'ils aimaient bien venir en français, que c'était bien, qu'on rigolait bien et qu'on apprenait des choses, donc ça fait du bien parfois! »

Sarah (Français) apprécie leur intérêt face à certaines propositions. Elle constate que sa meilleure connaissance des élèves, de leurs besoins mais aussi de leurs progrès, favorise leurs apprentissages : «créer le déclic, réussir à les mettre au travail » sont donc des défis qui, lorsqu'ils sont relevés, donnent aux débutants de sentiment d'être eux-mêmes en réussite dans une mission complexe et exigeante. Cet ensemble d'éléments conforte l'enseignant dans son rôle et sa légitimité, renforçant ainsi l'estime de soi.

A travers le récit de moments jugés réussis, on comprend combien le retour des élèves peut se transformer en ressource psycho-affective qui donne à l'enseignant l'envie de progresser dans 
Perez-Roux, T et Lanéelle, X. (2015). Pluralité des ressources pour l'action dans les processus de transition formation-emploi chez les enseignants du secondaire. Les Sciences de l'Education pour l'ère nouvelle, 48(4), pp. 17-40. https://www.cairn.info/revue-les-sciences-de-1-education-pour-1-ere-nouvelle-2015-4-p-17.htm

son activité au quotidien : «tous ils participaient, ils me posaient des questions. On a fait une question rapide en début de cours sur une factorisation et une élève qui a des difficultés m'a dit «madame, madame, on en refait une ? » parce qu'elle avait compris et qu'elle voulait réinvestir. Enfin, ils participaient bien, c'était vraiment très agréable... En plus, c'était des élèves généralement en difficultés qui, là, étaient très intéressés par ce qui se passait »(Julie, Mathématiques)

Les enseignants d'EPS mettent aussi fortement en relief cette dimension : «moi j'essaie d'être originale, d'avoir des entrées ludiques surtout pour les mettre dans l'activité et ça, ils aiment bien ! Avec mes $4^{\text {ièmes }}$ en saut de cheval, on était beaucoup sur la prise de risque, et c'était hyper intéressant. Ils sont entrés dans l'activité alors qu'il y avait des répétitions qui auraient pu les lasser mais non, ils faisaient des choses nouvelles, ils évoluaient. Ils ont vu leur progression du début jusqu'à la fin, donc c'était intéressant » (Fanny).

Ces énoncés, congruents avec les réponses au questionnaire, laissent à penser que diverses instances de légitimation participent à la construction identitaire des enseignants débutants. Le triptyque «devenir capable, être reconnu, se reconnaître »(Perez-Roux, 2012b) joue ici à plusieurs niveaux fortement reliés : se voir progresser et se sentir légitimé, être reconnu et se reconnaître comme intégré dans une communauté enseignante (regard des, appuis sur et travail avec les collègues) tout en s'affirmant progressivement, notamment à partir du regard des élèves (et des parents), comme un enseignant ayant une singularité, une manière-d'être-au-métier (Peyronnie, 1998) qui renforce l'identité et peut ouvrir sur le développement de nouvelles compétences. En ce sens, les élèves constituent, in fine, une ressource pour l'action.

\section{Discussion}

\subsection{Transition formation-emploi et mobilisation des ressources : complexité et dynamique}

Comme l'évoquent Dupuy et Le Blanc (2001), la transition génère un ensemble de turbulences appréhendées ici à partir des ressources mobilisées pour faire face aux changements et s'insérer dans un univers professionnel. Les résultats de l'enquête tendent à montrer la mobilisation de ressources plurielles et combinées. Certaines sont facilement repérables, d'autres fonctionnent comme des étayages discrets, difficiles à nommer mais participant d'un équilibre général du néo-professionnel. Ainsi, les ressources matérielles (ouvrages, logiciels, sites internet, etc.) constituent des points d'appui pour préparer l'action, pour mettre en perspective objectifs, contenus, démarches, modalités d'évaluation. Mais dans l'exercice du métier, dans les mises en œuvre, ce sont aussi des ressources relationnelles et humaines (collègues, élèves, personnes de confiance dans ou hors de l'établissement) qui sont mobilisées. Enfin, face à l'ampleur de la tâche qui met en jeu un processus d'enseignement/apprentissage complexe, face aux réussites partielles ou aux défis que posent les élèves, ce sont des ressources psychologiques (estime de soi, confiance, capacité à s'engager dans l'action) qui servent de soubassement pour construire progressivement une professionnalité enseignante. Une autre lecture est possible : dans cette dynamique des commencements, les ressources indirectes, institutionnelles (textes, programmes, inspection, politiques de formation), organisationnelles (logiques d'établissement, équipes disciplinaires, alternance) entrent en synergie avec des ressources personnelles, liées à l'histoire des individus, à leurs idéaux professionnels, à leur lecture singulière du monde scolaire et de ses acteurs, à la place qu'ils souhaitent y prendre.

De plus, les résultats montrent des articulations subtiles entre ressources et contraintes qui évoluent au fil du temps et des contextes d'intervention. Autrement dit, les ressources peuvent se construire chemin faisant, après avoir pu, même partiellement, revisiter autrement ce qui faisait obstacle. Le rapport aux élèves rend compte de cette problématique. Les attitudes des élèves vis-à-vis du travail constituent souvent une énigme pour les néo-enseignants (Perez-Roux 
Perez-Roux, T et Lanéelle, X. (2015). Pluralité des ressources pour l'action dans les processus de transition formation-emploi chez les enseignants du secondaire. Les Sciences de l'Education pour l'ère nouvelle, 48(4), pp. 17-40. https://www.cairn.info/revue-les-sciences-de-1-education-pour-1-ere-nouvelle-2015-4-p-17.htm

et Troger, 2011). Ces phénomènes se situent au cœur des tensions rencontrées lors de l'entrée dans le métier : surprises en termes de désajustement par rapport au prévu, malentendus liés au contrat didactique, désengagement des élèves face au travail demandé, etc. En même temps, ces élèves (ou d'autres) peuvent devenir des ressources: signaux d'adhésion, marques de reconnaissance, incidences des progrès réalisés donnent alors aux enseignants un sentiment de réussite et des leviers pour l'action. Plus largement, ils (ré)organisent le sens du métier, renvoyant au besoin de se sentir utile déjà repéré par Honneth (2000) comme un des axes de la reconnaissance au travail. Cette forme de reconnaissance concerne la manière dont les activités individuelles participent au bien de la société ; en ce sens, elle influe sur l'estime de soi, soutient la conviction d'une fonction sociale de l'activité, elle-même portée par le sentiment d'être utile. Un mouvement semble donc s'opérer des étayages externes vers des appuis internes au sujet. Il participe d'un processus d'appropriation qui renvoie aux conditions d'émergence de la professionnalité (Jorro et de Ketele, 2011)

\subsection{Le paradoxe des ressources construites en/par la formation : continuités, ruptures, perspectives}

Les résultats mettent aussi en avant une formation relativement critiquée par les FS : insuffisante durant le temps à l'université (un seul un FS sur 160 fait référence aux cours dispensés par le volet universitaire du master), mal calibrée pendant l'année de titularisation. Pourtant, à la question : «quels seraient vos souhaits en termes d'accompagnement professionnel futur ? » (2 réponses possibles parmi 5), «participer à des actions de formation continue » obtient 54\% des réponses. Deux autres items renforcent ce qui est relevé comme important dans l'année en cours : « avoir des réunions informelles avec les pairs » $(44 \%)$, «pouvoir bénéficier d'un tutorat prolongé » $(24 \%)$ et «avoir des réunions fréquentes avec l'inspection » $(20 \%)$, «participer à des réunions institutionnelles avec les pairs » (17\%). Il semble donc que l'essentiel soit de ne pas rester isolé dans un métier où les ressources peuvent s'épuiser, où la dimension humaine peut apporter réussites et déstabilisations, où le recul se révèle nécessaire et où la réflexivitédistanciation s'exerce sans doute mieux à plusieurs. Par ailleurs, la demande de travail avec les inspecteurs peut être interprétée comme un souci de réassurance, dans une année où les FS seront validés et où certains d'entre eux reprochent aux formateurs de ne pas donner suffisamment de réponses concrètes et applicables. Le paradigme du praticien réflexif (Schön, 1983) apparaît ainsi relativement éloigné des préoccupations des débutants : savoir ce qu'il y a à faire, avoir des réponses précises et définitives serait-il plus sécurisant ? L'affirmer n'occulte pas le paradoxe : au fil des entretiens, chacun revendique une part de bricolage, un rapport singulier au métier, un style d'enseignement (entrées ludiques par exemple) et, de fait, met en avant l'importance de la liberté pédagogique. Finalement, chacun tente, à sa manière, une orchestration des atouts et des faiblesses pour s'adapter au contexte et construire des repères qui pourront servir l'année suivante.

\section{Conclusion}

Les résultats mettent en lumière un système de ressources (mais aussi de contraintes) mobilisant normes institutionnelles relayées par la formation, cultures professionnelles plus ou moins partagées (travail en équipe, projets, mutualisation de documents, etc.) et formes de soutien dans lesquelles le rapport au tuteur prend une place essentielle. On constate que "l'idéal du métier » opère comme une ressource parfois coûteuse mais nécessaire pour orienter l'activité. Les élèves, malgré leur capacité à dérouter le néo-enseignant, constituent aussi une ressource essentielle : réussir à susciter l'intérêt, faire progresser certains élèves donne sens au métier, ouvre sur une forme de reconnaissance et renforce le sentiment de légitimité. On note enfin une tension entre 
Perez-Roux, T et Lanéelle, X. (2015). Pluralité des ressources pour l'action dans les processus de transition formation-emploi chez les enseignants du secondaire. Les Sciences de l'Education pour l'ère nouvelle, 48(4), pp. 17-40. https://www.cairn.info/revue-les-sciences-de-1-education-pour-1-ere-nouvelle-2015-4-p-17.htm

les apports de la formation initiale (connaissances disciplinaires, soutien à la conception de l'enseignement) et ceux de la formation continue ; dans cet espace, la pertinence des contenus abordés est jugée à l'aune des urgences à assumer. Ces éléments interrogent la pertinence des dispositifs de formation pour les acteurs en charge de l'accompagnement des néo-enseignants.

Finalement, cette étude éclaire les enjeux de la (pré)professionnalisation des enseignants du secondaire. L'universitarisation resterait-elle, aux yeux des étudiants, un lieu d'acquisition de savoirs savants et relativement formels préalables à de véritables mises en situation professionnelles? Le temps d'insertion professionnelle conduirait-il à un éloignement progressif des acquis de la formation initiale en privilégiant alors une professionnalisation par l'action? Elle invite désormais à une réflexion sur les nouveaux masters de l'enseignement mis en œuvre à la rentrée 2013 dans le cadre des ESPE.

\section{Bibliographie}

BUCHETON D. (Dir.). L'agir enseignant : des gestes professionnels ajustés. Toulouse : Octares, 2009.

DUPUY R. \& LE BLANC A. Enjeux axiologiques et activités de personnalisation dans les transitions professionnelles. Connexions, 2001, n $76(2)$, pp. 61-79.

Guibert P., LAZUeCh G. \& Rimbert, F. Enseignants débutants : faire ses classes. Rennes : PUR, 2008.

Hetu J.-C., Lavoie M. \& Baillauques S. (Dir.). Jeunes enseignants et insertion professionnelle. Bruxelles : De Boeck, 1999.

HONNETH A. La lutte pour la reconnaissance. Paris : Cerf, 2000.

JORRO A. \& DE KETELE J-M. La professionnalité émergente : quelle reconnaissance?

Bruxelles : De Boeck, 2011.

LANEELlE X. \& PEREZ-RouX T. (sous presse). Entrée dans le métier des enseignants et transition professionnelle : impact des contextes de professionnalisation et dynamiques d'acteurs. L'Orientation Scolaire et Professionnelle, 2014, n43 (4).

LANEELLE X. Le développement professionnel des néo-enseignants de l'enseignement primaire : contextes d'insertion, mobilisation et élaboration de ressources, Ressources, 2012, $\mathrm{n}^{\circ} 16$, pp. $117-125$.

Perez-Roux T. \& Troger V. Place des élèves et de leur réussite scolaire dans la construction de la professionnalité chez les futurs professeurs de lycée professionnel. Carrefours de l'Education, 2011, n³2, pp. 149-166.

PeREZ-RouX T. \& LANEelle X. La inserción profesional de los docentes en Francia: preocupaciones dominantes y transformaciones identitarias, Revista Española de Educación Comparada, 2012, $\mathrm{n}^{\circ} 20, \mathrm{pp} .325-354$.

PEREZ-RouX T. Les enseignants néo-titulaires à l'épreuve du métier : entre désenchantement et formes d'adaptation provisoires. Les Langues Modernes, 2006, n³, pp. 34-44.

PEREZ-Roux T. Construire une professionnalité enseignante à l'heure des réformes : enjeux de et défis pour - la formation. In PEREZ-ROuX $\mathrm{T}$ (Dir.). La professionnalité enseignante : modalités de construction en formation. Rennes : PUR, 2012a, pp. 97-120.

PEREZ-RouX T. Construction identitaire des enseignants débutants: quelle reconnaissance d'autrui pour se (re)connaître en tant que professionnel ? Recherches et éducations, $2012 \mathrm{~b}, \mathrm{n}^{\circ} 7$, pp.69-84.

PEYRONNIE H. Instituteurs : des maîtres aux professeurs d'école. Formation, socialisation et manière d'être au métier. Paris : PUF, 1998. 
Perez-Roux, T et Lanéelle, X. (2015). Pluralité des ressources pour l'action dans les processus de transition formation-emploi chez les enseignants du secondaire. Les Sciences de l'Education pour l'ère nouvelle, 48(4), pp. 17-40. https://www.cairn.info/revue-les-sciences-de-l-education-pour-1-ere-nouvelle-2015-4-p-17.htm

RAYOU P. \& VAN ZANTEN A. Enquête sur les nouveaux enseignants. Changeront-ils l'école? Paris : Bayard, 2004.

SCHÖN D-A. The Reflexive Practitioner. How Professionals Think in Action, États-Unis : Basic Book Inc., 1983.

Wentzel B., AKkari A., CoEn PF. \& ChangKakoti N. (Dir.). L'insertion professionnelle des enseignants : regards croisés et perspective internationale. Bienne : Editions HEP-BEJUNE, 2011.

WitTORSKI R. \& BRIQUET-DUHAZE S. (Dir.). Comment les enseignants apprennent-ils leur métier? Paris : L'Harmattan, 2008.

Zittoun T. \& Perret-Clermont A-N. Contributions à une psychologie de la transition. Congrès international de la Société suisse pour la recherche en éducation (SSRE), 2001. 